\title{
A decent proposal
}

\section{Luis C. Corchón ${ }^{1}$, Carmen Herrero ${ }^{2}$}

1 Universidad Carlos III, Departamento de Economía, c/Madrid 126, 28903 Getafe (Madrid) Spain (e-mail: 1corchon@eco.uc3m.es)

2 Universidad de Alicante and IVIE, 03071 Alicante, Spain

(e-mail: carmen.herrero@ua.es)

\begin{abstract}
In this paper we explore the notion that players are "decent" in the sense that their choices are bounded by certain unwritten social rules. We apply this idea to problems of Bankruptcy and Implementation.
\end{abstract}

JEL Classification: D78, D63

Key words: Fairness constraints, implementation, bankruptcy, bargaining

\section{Introduction}

Some years ago, models based on the neoclassical tradition assumed that people always behaved according to their true preferences. Then came the incentives point of view, and agents were assumed to cheat and to take any possible advantage as long as it was profitable.

In this paper we attempt to model situations that lie between complete honesty and total dishonesty: We assume that agents act strategically but that their behavior is bound by certain self-imposed rules. "Norms of behavior entail ... self-imposed standards of conduct, such as honesty and integrity", North (1989). ${ }^{1}$ An example

The authors wish to express their gratitude for comments and suggestions to N. Baigent, S. Baliga, V. Bashkar, C. Beviá, S. Chattopadhayay, G. Charness, M. Maschler, C. Matutes, P. McAfee, I. OrtuñoOrtín, A. Rangel, J. Roemer, J. Schummer, T. Sjöström, W. Thomson, the participants in seminars in Hakone (Japan), Harvard, Queen's University, Rochester, Studienzentrum at Gergenzee (Switzerland), two anonymous referees and an associate editor. The usual caveat applies. This research has been partially supported by grants BEC2002-02194, BEC2001-0535, and GV 01-371. This paper is dedicated to Bob Aumann, from whom we have learned so much.

1 Many psychologists believe that one's conscience does not always serve one's self-interest (see Prelec and Herrstein 1991). Also, the internalization of rules of decent behavior has been invoked as a way of explaining violations of the principle of rational choice. See Sen $(1983,1993)$. 
of this might be a departmental meeting where some painful duty (e.g., service) is assigned among faculty members: A proposal like "I am not willing to do anything for the department" is rarely made. Instead, each agent looks for the best proposal that she can put on the table without being accused of being "indecent". Another example is the following: Suppose that the budget of a university is reduced. The dean, after hearing the chairmen, makes a decision. ${ }^{2}$ Anticipating that the dean will try to average the losses, a chairman might ask for more than she had before the reduction. But this strategy may not work (and even may harm her cause) because such a demand would be dismissed on the grounds of not being decent. In other words, proposals must meet certain public standards in order to be taken seriously.

The impact of these constraints is well explained in the words of a historian: “... the costs of measurement and enforcement of contracts ... offer ample opportunity for antisocial behavior; and without rules being supplemented by self-imposed standards of conduct which constrain maximization at some margins it is hard to believe that such complex societies would be viable. But the economic models we employ have little room for such behavioral complexity. Trust, ethical standards of conduct, and moral precepts do influence the cost of contracting and the performance of economies... A major challenge to the social scientist is to develop politicaleconomic models that are institutionally rich and can take into account more complex behavior than has been done heretofore", North (1989). ${ }^{3}$ Also, Shapin (1994) explains how in seventeen century England, decency requirements shaped the form of modern science in its beginnings: "Assessments of the reliability of empirical narratives.... were.. founded upon.... "natures", "temperaments" and "dispositions" of different types of people", and that "gentleman... faced a pragmatic consideration that enjoined them to be... reliable truth-tellers" (p. 78).

In this paper we analyze the impact of decency constraints on resource allocations but we do not address the question of why they are binding. We feel that it would be unfair to dismiss our approach because it does not provide foundations for the idea of why decency matters. After all, models of price-taking agents were used for many years before foundations for this behavior were provided. The same argument applies to the postulate that agents are rational. ${ }^{4}$

What are these decency constraints? This is a difficult question because these constraints can take two forms: On the one hand, there are allocations that are not decent (i.e., the dictatorial allocation). On the other hand, in a given mechanism, there are messages that are not decent, like to falsely accuse somebody of cheating when one is cheating himself. Thus, decency constraints depend on the particular mechanism that is being used. All we can say, at this stage, is that "decent behavior" can be defined in a natural way in some instances: The model of bankruptcy, O'Neill

\footnotetext{
2 Other possibilities are that the dean computes directly what she thinks is a fair solution or that agents submit allocation rules that determine the allocation rule that is used (see Van Damme 1986; Chun 1989; Anbarci and Yi 1992; Herrero 2003).

3 The importance of decency in politics is epitomized by the sentence "Don't you have any decency?", which ended an era of modern American history.

4 At this point it may be useful to remark that, according to Adam Smith, human beings have two kind of sentiments: "self-interest" and "benevolence". It may well be that decent behavior is just a reflection of benevolence.
} 
(1982), Aumann and Maschler (1985), the non-cooperative model of bargaining in exchange economies, and the canonical mechanism in Nash Implementation, Jackson (2001). The aim of this paper is not to make an exhaustive analysis of such cases, but to show that the combination of the ideas of decency and rationality can produce analytical results (see Rabin 1993 for a similar attempt).

The bankruptcy and bargaining models have similar structures: Agents make demands that must lie in a certain set in order to be considered as being decent. In the bankruptcy model no agent can demand more that her claim. In the bargaining model no agent can propose an allocation that is not individually rational for the other agent. Decent proposals are converted into feasible allocations by a compromise function (bankruptcy) or an outcome function (bargaining). A Decent Dominant Strategy $(D D S)$ for an agent is a decent proposal that, for any decent proposals made by other agents, is better for her than any other decent proposal she can make. A Nash Equilibrium in Decent Strategies (NEDS) is a list of decent proposals such that no agent can increase her utility through a decent proposal.

In the canonical model of Nash Implementation, a message is a triple $=($ an economy, an allocation, an integer) (an economy is a description of the preferences). We impose the following requirements on the set of decent messages: If all agents announce (the true economy, an allocation prescribed by the SCC, one), this list of messages is decent. If all the other agents minus, say, $i$, send the same message, it is decent for $i$ to state (the true economy, an allocation, one) if the allocation decreases the utility of $i$ according to the report made by the others. Finally, if at least two agents disagree on their reports, and it is possible for another agent to increase her utility by means of a certain message, there is a decent message in which she can do so. This message may imply very modest gains in utility terms. This contrasts with the canonical mechanism, in which agents may get what they ask for, including the whole bundle of goods available!. Later on we consider a stronger decency requirement.

The main results of this paper are: In Sect. 2 we show that a solution to the bankruptcy problem can be implemented in DDS if and only if what any agent receives is strictly increasing on her claim. This implies that, for the case of three or more agents, the Proportional or the Random Arrival solutions are implementable in DDS but that the Constrained Equal Awards, the Constrained Equal Losses or the Talmud solutions are not. Later on, we also consider what can be implemented by simple mechanisms. We find a stark contrast between the cases of two and more than two agents. In Sect. 3 we show that in the bargaining model with transferable utility, the Shapley value (which in this domain coincides with other solutions - Nash, Raiffa, Kalai-Smorodinsky, etc. -) can be implemented in NEDS. We also show that, in a large class of environments, including exchange economies with more than three agents, if certain social goals can be implemented by means of a Nash Equilibrium (NE), they can be implemented by means of NEDS and, with a stronger decency requirement, that any selection of the Pareto efficient correspondence is implementable in NEDS. Since the Shapley value and some selections of the Pareto efficient correspondence cannot be implemented in NE, our results show that the consideration of decency constraints enlarges the possibility of implementing social goals. In Sect. 4 our final comments are presented. 


\section{Decent implementation of solutions to the bankruptcy problem}

Suppose $n$ agents, each one having claims on a specific amount of money, $c_{i}$, $i=1, \ldots, n$. The total amount at hand $(E)$ might not be enough to cover all the claims. An economy is a pair $e=(E, c) \in \mathbb{R}_{++}^{n+1}$, where $c=\left(c_{1}, \ldots, c_{n}\right)$. Let $\mathbb{E}$ be the family of economies such that for all $e=(E, c) \in \mathbb{E}, c_{i} \leq E,{ }^{5}$ for all $i=1, \ldots, n$. Agents always prefer to receive more money. Thus, in this section, preferences are fixed. Let $C=c_{1}+\cdots+c_{n}$.

We shall assume that $\mathbb{E}$ is claims-comprehensive, ${ }^{6}$

$$
\text { If } e=(E, c) \in \mathbb{E} \text {, and } e^{\prime}=\left(E, c^{\prime}\right) \text {, with } c^{\prime} \leq c \text {, then } e^{\prime} \in \mathbb{E} \text {. }
$$

Let us look for reasonable rules for dividing the estate $E$ among the claimants. These rules will be called solutions. Formally, a solution is a function $\Phi: \mathbb{E} \rightarrow \mathbb{R}_{+}^{n}$ such that for all $e=(E, c) \in \mathbb{E}, \Phi(e) \leq c$ and $\Phi_{1}(e)+\cdots+\Phi_{2}(e)=\min \{C, E\}$. This means that if $C \leq E$, then $\Phi(e)=c$.

We assume that no solution can be achieved directly because agents know claims but the planner does not. Thus, the planner asks agents about their claims. Formally:

A strategy (message) for agent $i, i=1, \ldots, n$, is a pair of real numbers $m_{i}=\left(m_{i i}, M_{i}\right)$, where $m_{i i}$ is what agent $i$ asks for himself, and $M_{i}$ is the total amount to be distributed according to $i$. A strategy profile is a tuple $m=\left(m_{1}, \ldots, m_{n}\right)$. Let $S=\mathbb{R}^{2 n}$ be the set of strategy profiles. We will use the following notation: for any pair of strategy profiles, $m, \widetilde{m} \in S$, $\left(\widetilde{m}_{i}, m_{-i}\right)=\left(m_{1}, \ldots, m_{i-1}, \widetilde{m}_{i}, m_{i+1}, \ldots, m_{n}\right) \in S$.

In this framework, our definition of a decent strategy is that no one asks for herself more than her claim. Formally:

A strategy profile $m=\left(m_{1}, \ldots, m_{n}\right) \in S$ is decent in economy $e=(E, c)$, if for all $i=1, \ldots, n$ we have $0 \leq m_{i i} \leq c_{i}$, and $M_{i}=\min \{E, C\}$. Let us denote the set of decent strategies in $e$ by $D(e)$.

As an example, suppose that the dean of a university has to allocate some money to cover teaching whose exact quantity is unknown to her. Suppose that the dean asks each department to submit its needs for teachers. When the chairman of a department decides about the number of teachers to be asked, she might restraint herself from asking too much because if this were the case, her behavior might be termed "greedy" from her fellow chairmen. And bad reputation has unknown consequences, from mere gossip to a public inquiry. Similarly, when submitting a $\mathrm{CV}$ in order to get grants, scholarships, etc., people seldom falsify their publication achievements or their existing computers, machines, etc, when CV and existing equipment act as a claim. Accordingly, committees in charge of awarding grants

5 This restriction puts some 'natural' limits on individual claims, making the analysis of different solutions to the bankruptcy problem easier, avoiding distinctions between game-theoretical and nongame-theoretical solutions. See Dagan and Volij (1993).

6 Note that claims-comprehensiveness implies that in $\mathbb{E}$ we have not only "normal" bankruptcy problems, but also "feasible" problems for which $c_{1}+c_{2} \leq E$. 
very rarely inquire about the information provided about the claims and, instead, trust that the behavior of agents is decent.

We will assume that once the agents have made their proposals, a decision will be reached. Such a decision can be made by a real person, or it might reflect how agreements are reached among people living in a community. We formalize this notion by means of the concept of a compromise function.

A compromise function is a continuous mapping $F: S \rightarrow \mathbb{R}_{+}^{n}$ such that for all $e=(E, c)$ and for all $m \in D(e), F_{1}(m)+\cdots+F_{n}(m) \leq \min \{E, C\}$.

Let us now model the strategic behavior of the agents.

The Solution $\Phi$ is implementable in Decent Dominant Strategies $(D D S)$ if there exists a compromise function $F$ such that for all $e=(E, c) \in \mathbb{E}$, there exists $m^{*}(e) \in D(e)$ such that:

(i) $\Phi(e)=F\left[m^{*}(e)\right]$

(ii) For all $i=1, \ldots, n$, for all $e \in \mathbb{E}$, and for all $m \in D(e), F_{i}\left(m_{i}^{*}(e), m_{-i}\right) \geq$ $F_{i}\left(m_{i}, m_{-i}\right)$, with strict inequality if $m_{i i}^{*}(e) \neq m_{i i}$.

There are two extra requirements in addition to the usual ones in implementation: The Dominant Strategy must be unique and the compromise function must be continuous. Let us discuss these points in turn. There are three reasons why we insist on the inequality in (ii) above being strict if $m_{i i} \neq m_{i i}^{*}(e)$. First, it gives the agents real incentives to choose the equilibrium strategy, since any deviations from it will hurt the welfare of the deviating agent. Second, it guarantees that there are no other equilibria than the one yielding the required allocation. In particular, it precludes the existence of Nash Equilibria yielding allocations different from the one prescribed by $\Phi$. Finally, being regarded as a Nash Equilibrium, it is strict, so that it passes all known refinements. We also require continuity of the compromise function because we do not want small changes in the messages to be translated into large changes in the resulting allocation. We now present our first result:

Proposition 1. Let $\Phi$ be a continuous solution such that for all $i=1, \ldots, n, \Phi_{i}$ is strictly increasing on $c_{i}$. Then, $\Phi$ is implementable in decent dominant strategies.

Proof. We shall limit the definition of our compromise function $F$ to those strategies that are decent for some $e \in \mathbb{E}$. For all $e \in \mathbb{E}$, and for all $m \in D(e)$, let us call $c^{\prime}=\left(m_{11}, \ldots, m_{n n}\right)$, and $E^{\prime}=M_{1}=\cdots=M_{n}$. Let $e_{m}=\left(E^{\prime}, c^{\prime}\right)$. By claims-comprehensiveness, $e_{m} \in \mathbb{E}$. Then, define $F(m)=\Phi\left(e_{m}\right) . F$ is trivially a compromise function.

Let us now show that such $F$ implements $\Phi$ in DDS:

Case 1. $C>E$. Take $m^{*}(e)=\left(m_{1}^{*}(e), \ldots, m_{n}^{*}(e)\right)$, where $m_{i i}^{*}(e)=c_{i}$, and $M_{i}^{*}(e)=E$, for all $i=1, \ldots, n$. By construction, $F\left[m^{*}(e)\right]=\Phi\left(e_{m^{*}(e)}\right)=\Phi(e)$. Moreover, for all $m \in D(e)$, we have that $F_{i}(m)=\Phi_{i}\left(e_{m}\right) \leq \Phi_{i}\left(e_{\left(m_{i}^{*}(e), m_{-i}\right)}\right)=$ $F\left(m_{i}^{*}(e), m_{-i}\right)$, with an strict inequality if $m_{i i} \neq m_{i i}^{*}(e)$ due to $\Phi_{i}$ be strictly increasing with respect to $c_{i}$.

Case 2. $C \leq E$. Take $m^{*}(e)=\left(m_{1}^{*}(e), \ldots, m_{n}^{*}(e)\right)$, where $m_{i i}^{*}(e)=c_{i}$, and $M_{i}^{*}(e)=C$, for all $i=1, \ldots, n$. Again, $e_{m^{*}(e)}=e$, and thus, $F\left[m^{*}(e)\right]=$ $\Phi\left(e_{m^{*}(e)}\right)=\Phi(e)$. Moreover, for all $m \in D(e), F_{i}(m)=\Phi_{i}\left(e_{m}\right) \leq$ 
$\Phi_{i}\left(e_{\left(m_{i}^{*}(e), m_{-i}\right)}\right)=F\left(m_{i}^{*}(e), m_{-i}\right)$, with a strict inequality if $m_{i i} \neq m_{i i}^{*}(e)$ due to $\Phi_{i}$ being strictly increasing with respect to $c_{i}$.

In order to obtain a converse of Proposition 1, we first present the following lemmata:

Lemma 1. Let $\Phi$ be a solution implementable in decent dominant strategies. Then $\Phi_{i}$ is weakly increasing on $c_{i}$, for all $i=1, \ldots, n$.

Proof. Let $c_{i}<c_{i}^{\prime}, e=(E, c), e^{\prime}=\left(E, c^{\prime}\right) \in \mathbb{E}$, where $c^{\prime}=\left(c_{i}^{\prime}, c_{-i}\right)$. Since $\Phi$ is implementable in DDS, there exist $m^{*}(e) \in D(e), m^{*}\left(e^{\prime}\right) \in D\left(e^{\prime}\right)$ such that $F\left[m^{*}(e)\right]=\Phi(e), F\left[m^{*}\left(e^{\prime}\right)\right]=\Phi\left(e^{\prime}\right)$.

Consider the following cases:

Case 1. $C>E$. Thus, $C^{\prime}>E$, and $F_{i}\left[m_{i}^{*}\left(e^{\prime}\right), m_{-i}^{*}\left(e^{\prime}\right)\right] \geq F_{i}\left[m_{i}^{*}(e), m_{-i}^{*}\left(e^{\prime}\right)\right]$, because $\left[m_{i}^{*}(e), m_{-i}^{*}\left(e^{\prime}\right)\right] \in D\left(e^{\prime}\right)$.

Now, let $j \neq i$. Assume that $m_{j j}^{*}(e) \neq m_{j j}^{*}\left(e^{\prime}\right)$. Thus, we have $F_{j}\left[m_{j}^{*}\left(e^{\prime}\right), m_{-i}^{*}(e)\right]>F_{j}\left[m_{j}^{*}(e), m_{-i}^{*}(e)\right]$, because $m^{*}(e) \in D\left(e^{\prime}\right)$. Additionally, $F_{j}\left[m_{j}^{*}(e), m_{-i}^{*}(e)\right]>F_{j}\left[m_{j}^{*}\left(e^{\prime}\right), m_{-i}^{*}(e)\right]$, because $\left[m_{j}^{*}\left(e^{\prime}\right), m_{-i}^{*}(e)\right] \in$ $D(e)$. Contradiction, and consequently, for all $j \neq i, m_{j j}^{*}(e)=m_{j j}^{*}\left(e^{\prime}\right)$. As a consequence, and since $M_{j}^{*}(e)=M_{j}^{*}\left(e^{\prime}\right)=E$, we have that $m_{j}^{*}(e)=$ $m_{j}^{*}\left(e^{\prime}\right)$. Thus, we have $\Phi_{i}\left(e^{\prime}\right)=F_{i}\left[m_{i}^{*}\left(e^{\prime}\right), m_{-i}^{*}\left(e^{\prime}\right)\right] \geq F_{i}\left[m_{i}^{*}(e), m_{-i}^{*}\left(e^{\prime}\right)\right]=$ $F_{i}\left[m_{i}^{*}(e), m_{-i}^{*}(e)\right]=\Phi_{i}\left(e^{\prime}\right)$, with a strict inequality if $m_{i i}^{*}\left(e^{\prime}\right) \neq m_{i i}^{*}(e)$.

Case 2. $C^{\prime} \leq E$. Thus, $C<E$. Because of the implementability hypothesis, $\Phi_{i}(e)=c_{i}<c_{i}^{\prime}=\Phi_{i}\left(e^{\prime}\right)$.

Case 3. $C \leq E<C^{\prime}$. Because of the implementability hypothesis, $\Phi_{i}(e)=$ $F_{i}\left[m^{*}(e)\right]=c_{i}$. Now, a similar reasoning to that in Case 1 allows us to obtain the result.

Lemma 1 is a sort of revelation principle for decent dominant implementation. Note that it states that whenever $\Phi$ is implementable in DDS, for all $c_{-i}, E, c_{i}$, it follows that $\Phi_{i}\left(c_{i}, c_{-i}, E\right) \geq \Phi_{i}\left(c_{i}^{\prime}, c_{-i}, E\right)$, for all $c_{i}^{\prime}<c_{i}$. In other words, revealing $c_{i}$ is a dominant strategy for agent $i$ in the direct revelation mechanism. We now show that continuity of the compromise function $F$ implies that $\Phi_{i}$ is strictly increasing, that is, in the direct revelation mechanism, revealing $c_{i}$ is the only dominant strategy for agent $i$.

Lemma 2. Let $\Phi$ be a solution implementable in DDS. Then, $\Phi_{i}$ is strictly increasing on $c_{i}$, for all $i=1, \ldots, n$.

Proof. Suppose not. Then there exists $c_{-i}, E$, and an interval $\left[\underline{c}_{i}, \bar{c}_{i}\right]$ such that for all $c_{i} \in\left[\underline{c}_{i}, \bar{c}_{i}\right], \Phi_{i}\left(c_{i}, c_{-i}, E\right)=k$, while if $c_{i}^{\prime}>\bar{c}_{i}, \Phi_{i}\left(c_{i}^{\prime}, c_{-i}, E\right)>k$.

Because of the implementability hypothesis, for all $c_{i} \in\left[\underline{c}_{i}, \bar{c}_{i}\right]$, there exists $m_{i i}^{*}\left(c_{i}\right), m_{j j}^{*}\left(c_{j}\right), j \neq i$, such that $\Phi\left(c_{i}, c_{-i}, E\right)=F\left[m_{i i}^{*}\left(c_{i}\right), m_{-i i}^{*}\left(c_{-i}\right), E\right]$. Furthermore, $F_{i}\left[m_{i i}, m_{-i i}^{*}\left(c_{-i}\right), E\right]<F_{i}\left[m_{i i}^{*}\left(c_{i}\right), m_{-i i}^{*}\left(c_{-i}\right), E\right]=\phi_{i}\left(c_{i}, c_{-i}, E\right)=$ $k$, for all $m_{i i} \leq c_{i}, m_{i i} \neq m_{i i}^{*}\left(c_{i}\right)$.

Previous inequality indicates that for all $c_{i} \in\left[\underline{c}_{i}, \bar{c}_{i}\right], m^{*}\left(c_{i}\right)=d_{i} \leq \underline{c}_{i}$. Furthermore, $F_{i}\left[\bar{c}_{i}, m_{-i i}^{*}\left(c_{-i}\right), E\right]<k$. Let $\varepsilon=k-F_{i}\left[\bar{c}_{i}, m_{-i i}^{*}\left(c_{-i}\right), E\right]$. 
Since $F$ is continuous, there exists a $\delta>0$ such that whenever $\left|c_{i}-\bar{c}_{i}\right|<\delta$, then $\left|F_{i}\left[c_{i}, m_{-i i}^{*}\left(c_{-i}\right), E\right]-F_{i}\left[\bar{c}_{i}, m_{-i i}^{*}\left(c_{-i}\right), E\right]\right|<\varepsilon / 2$. Take now $c_{i}^{\prime}>\bar{c}_{i}$ such that $c_{i}^{\prime}-\bar{c}_{i}<\delta$. Thus, $\left|F_{i}\left[c_{i}^{\prime}, m_{-i i}^{*}\left(c_{-i}\right), E\right]-F_{i}\left[\bar{c}_{i}, m_{-i i}^{*}\left(c_{-i}\right), E\right]\right|<\varepsilon / 2$. And this holds for all $c_{i}^{\prime}$ fulfilling previous conditions.

Now, because of the implementability hypothesis, there exists $m_{i i}^{*}\left(c_{i}^{\prime}\right) \leq$ $c_{i}^{\prime}$ such that $\Phi_{i}\left(c_{i}^{\prime}, c_{-i}, E\right)=F_{i}\left[m_{i i}^{*}\left(c_{i}^{\prime}\right), m_{-i i}^{*}\left(c_{-i}\right), E\right]>k$. But then, $m_{i i}^{*}\left(c_{i}^{\prime}\right)>\bar{c}_{i}$, and thus, $F_{i}\left[m_{i i}^{*}\left(c_{i}^{\prime}\right), m_{-i i}^{*}\left(c_{-i}\right), E\right]-F_{i}\left[\bar{c}_{i}, m_{-i i}^{*}\left(c_{-i}\right), E\right]>$ $k-F_{i}\left[\bar{c}_{i}, m_{-i i}^{*}\left(c_{-i}\right), E\right]=\varepsilon$, which is a contradiction.

From Proposition 1 and Lemmas 1 and 2 we obtain the following:

Theorem 1. Let $\Phi$ be a continuous solution. Then $\Phi$ is implementable in decent dominant strategies iff $\Phi_{i}$ is strictly increasing on $c_{i}$, for all $i=1 \ldots, n$.

As a consequence of Theorem 1, the proportional $(P)$, or the random arrival $(R A)$ solutions are implementable in DDS, while the constrained equal awards $(A)$ is not. The Talmud $(T)$ and the constrained equal-loss $(L)$ solutions are implementable in DDS in the two person case, but they are not for three or more agents. ${ }^{7}$ Note that, since we do not allow claims to exceed the estate, it happens that, in the two person case,

$$
T_{i}(E, c)=L_{i}(E, c)=\frac{1}{2}\left(c_{i}-c_{j}+E\right)
$$

which are, indeed, strictly increasing on $c_{i}$.

We now look for mechanisms that are simple, so they can be easily understood without much explanation. The simplest form that $F($ ) can take is that depends on a single number and that this number is additively separable in the messages of the agents, i.e.

$$
F_{i}=\min \left\{c_{i}, \frac{m_{i i}+\sum_{j \neq i} \alpha_{j i}\left(M_{j}-m_{j j}\right)}{n}\right\}
$$

where $\alpha_{i j}$, are given parameters such that $0 \leq \alpha_{i j} \leq 1$, with $\sum_{j} \alpha_{i j}=1$. Clearly, the only DDS is $m_{i i}=c_{i}$. Consider, now, the two agents case. For $\alpha_{j i}=1$, $i, j=1,2$ we get that

$$
F=\frac{1}{2}\left[\left(c_{1}+E-c_{2}\right),\left(c_{2}+E-c_{1}\right)\right],
$$

${ }^{7}$ For the sake of completeness, we provide the definition of the most prominent solutions to the bankruptcy problem. See Thomson (1985)

Proportional. $P_{i}(E, c)=\lambda c_{i}$, where $\lambda$ is chosen so that $\sum P_{i}(E, c)=E$

Constrained Equal Awards $A_{i}(E, c)=\min \left\{c_{i}, \lambda\right\}$, where $\lambda$ is chosen so that $\sum A_{i}(E, c)=E$

Constrained Equal Losses, $L_{i}(E, c)=x_{i}$, where $c_{i}-x_{i}=\max \{\lambda, 0\}$, and where $\lambda$ is chosen so that $\sum L_{i}(E, c)=E$

Talmud, $T_{i}(E, c)= \begin{cases}A_{i}(E, c / 2) & \text { if } E \leq C / 2 \\ c_{i} / 2+L_{i}(E-C / 2, c) & \text { otherwise }\end{cases}$

Random Arrival, $R A$, is the expected payoff obtained by all agents when all arrival orders are equally likely, and in each arrival agents are sequentially satisfied up to the available amount of money. 
and we obtain the so-called standard solution (which, in this case, and because of the restricted domain chosen, coincides with $T, R A$ and $L$ ). The standard solution, nonetheless, differs from $P$ and $A$. In this case, thus, the standard solution is implementable in DDS by a particularly simple compromise function, namely, the average of the proposals made by the agents. ${ }^{8}$

In the case $n>2$, and by calling $\Lambda=\left(\alpha_{i j}\right)_{j \neq i}$,

$$
\Phi_{i}^{\Lambda}(E, c)=\frac{1}{n}\left[c_{i}+\sum_{j \neq i} \alpha_{j i}\left(E-c_{j}\right)\right]
$$

We obtain

$$
F_{i}=\min \left\{c_{i}, \Phi_{i}^{\Lambda}(E, c)\right\}
$$

Note that all solutions in this family are implementable in DDS, since, for all $i=1, \ldots, n, \Phi_{i}^{\Lambda}$ (and thus, $F_{i}$ ) is strictly increasing on $c_{i}$. Nonetheless, for $n>2$, no solution in this family coincides with any of the solutions mentioned before.

Proposition 2. For $n>2$, no solution in the set $\{R A, T, P, A, L\}$ belongs to the family $\Phi^{\Lambda}$.

Proof. First notice that, for $n \geq 3, T_{i}, A_{i}$, and $L_{i}$ are not strictly increasing on $c_{i}$.

Let $E=6, c=(6,5,2), c^{\prime}=(6,5,2.5)$. Then, $A(E, c)=T(E, c)=$ $(2,2,2)=A\left(E, c^{\prime}\right)=T(E, c), L(E, c)=(3.5,2.5,0)=L\left(E, c^{\prime}\right)$.

Now, let $n=3$, we can parameterize $\Phi^{\Lambda}$ by means of six parameters, $\alpha_{i j}$, $i \neq j, i, j=1,2,3$, so that

$$
\begin{aligned}
& \Phi_{1}^{\Lambda}(E, c)=\frac{1}{3}\left[c_{1}+\alpha_{21}\left(E-c_{2}\right)+\alpha_{31}\left(E-c_{3}\right)\right] \\
& \Phi_{2}^{\Lambda}(E, c)=\frac{1}{3}\left[c_{2}+\alpha_{12}\left(E-c_{1}\right)+\alpha_{32}\left(E-c_{3}\right)\right] \\
& \Phi_{3}^{\Lambda}(E, c)=\frac{1}{3}\left[c_{3}+\alpha_{13}\left(E-c_{1}\right)+\alpha_{23}\left(E-c_{2}\right)\right]
\end{aligned}
$$

Let $E=6, c=(6,5,4)$. For this example, for all admissible values of $\Lambda$, it happens that $\Phi_{i}^{\Lambda} \leq c_{i}$, for all $i$.

$$
\begin{aligned}
& \Phi_{1}^{\Lambda}(E, c)=\frac{1}{3}\left[6+\alpha_{21}+2 \alpha_{31}\right] \\
& \Phi_{2}^{\Lambda}(E, c)=\frac{1}{3}\left[5+2 \alpha_{32}\right] \\
& \Phi_{3}^{\Lambda}(E, c)=\frac{1}{3}\left[4+2 \alpha_{23}\right]
\end{aligned}
$$

We obtain that $R A(E, c)=(7.5,2,4.5)$, and $P(E, c)=(2.4,2,1.6)$ cannot be obtained for any value of $\Lambda$. In both cases, $\alpha_{32}=1 / 2$, and thus, $\alpha_{31}=1 / 2$. For $R A(E, c)$, moreover, $\alpha_{23}>1$; For $P(E, c)$, we have that $\alpha_{23}=.4$, and then $\alpha_{21}=.6$, but then $\frac{1}{3}\left[6+\alpha_{21}+2 \alpha_{31}\right]=2.5333 \neq 2.4$.

Proposition 2 has two implications: One, positive, that, when $n>2$, there are solutions to the bankruptcy problem that are, both, new and implementable in

8 In the arbitration literature, there is a wide consensus that arbitrators tend to split-the-difference between the last offers of the parties (see Stevens (1966), or Farber (1981)). This result could be interpreted by saying that, in the two-agents case, the splitting-the-difference mechanism implements the standard solution in DDS.

This fact does not generalize to the $\mathrm{n} \geq 3$ agents case, as we see later. 
DDS by the simplest compromise function, namely, a linear one. Another is mainly negative. Our approach points out that the case $n=2$ and the case of $n>2$ are, somehow, different, a fact already encountered in other areas of economics, like mechanism design and dynamics. Unfortunately, current theories do not pay attention to this fact.

\section{A theory of decent implementation when preferences are unknown to the planner}

In this section, we develop a theory of implementation based on the notion that strategies must be decent. Otherwise, most of the concepts are borrowed from the theory of implementation.

Let $A$ be the set of feasible allocations (alternatives), assumed to be fixed. An economy, denoted by $e$, is a list of preferences defined over allocations. The space of economies is denoted by $E$. Preferences of agent $i$ are representable by a utility function $u_{i}: A \times E \rightarrow \Re$.

A Social Choice Correspondence (SCC) is a mapping $\phi: E \rightarrow A$. This mapping represents the allocations that the planner would like to achieve in each economy in $E$. But the wishes of the planner cannot be achieved directly because preferences are not verifiable. Hence, the need for a decentralized procedure to reach decisions, i.e., a mechanism.

A Mechanism is a list $\mathcal{M}=\left(M_{1}, \ldots, M_{n}, g\right)$ where $g: M \rightarrow A$ and $M \equiv$ $\times_{i=1}^{n} M_{i}$. Let $m$ be an element of $M$. Sometimes we will write $m=\left(m_{i}, m_{-i}\right)$. Define $u_{i}(a, e)=u_{i}(g(m), e) \equiv v_{i}(m, e)$. The list $\left(v_{1}(, e), \ldots, v_{n}(, e)\right)$ will be called the game (in normal form) associated to $(\mathcal{M}, e)$.

Given a mechanism $\mathcal{M}$ and an economy $e$, let $D(\mathcal{M}, e) \subseteq M$ be the set of decent messages. This set embodies two kinds of restrictions. On the one hand, we have the allocations that agents think are decent in a particular economy. Let $d_{i}(e) \subseteq A$ be the set of allocations that $i$ thinks are decent in economy $e$. Thus, if $a \notin d_{i}(e)$ for some $i$ and $g(\check{m})=a, \check{m} \notin D(\mathcal{M}, e)$. On the other hand, certain messages (i.e., to falsely accuse somebody of cheating, etc.) might be not decent, even if they yield decent allocations. It is possible to regard these restrictions as embodying certain cultural aspects of the society in which a mechanism is applied. Let $D_{i}\left(\mathcal{M}, e, m_{-i}\right) \equiv\left\{m_{i} \in M_{i} /\left(m_{i}, m_{-i}\right) \in D(\mathcal{M}, e)\right\}$ be the set of decent messages for $i$, given $m_{-i}$.

A vector of messages $\left(m_{1}^{*}, \ldots, m_{n}^{*}\right) \in D(\mathcal{M}, e)$ is a Nash Equilibrium in Decent Strategies (NEDS) for the game associated to $(\mathcal{M}, e)$ if

$$
v_{i}\left(m_{i}^{*}, m_{-i}^{*}, e\right) \geq v_{i}\left(m_{i}, m_{-i}^{*}, e\right), \forall m_{i} \in D_{i}\left(\mathcal{M}, e, m_{-i}^{*}\right), \forall i .
$$

In words, a NEDS is a list of decent messages such that, for every agent, her message is the best decent message, given the decent messages of the rest of the agents. Let $D N S(\mathcal{M}, e)$ be the set of strategies that are a NEDS for the game associated with $(\mathcal{M}, e)$.

A SCC $\phi$ is implementable in NEDS if there is a mechanism $\mathcal{M}$ such that $\forall e \in E, D N S(\mathcal{M}, e) \neq \emptyset$ and $g(D N S(\mathcal{M}, e))=\phi(e)$. 
What is the relationship between Nash Implementation and NEDS Implementation? Recall from the above definition that implementation has two parts: Part 1 is to "sustain" allocations in $\phi(e)$ by means of an equilibrium. Part 2 is that there cannot be equilibria yielding allocations outside $\phi(e)$. It is clear that, in general, if it is possible to sustain an allocation by means of a Nash Equilibrium (NE), this allocation is also sustainable as a NEDS. But in Part 2 we have that there might be NEDS that are not NE. As a result, it is not possible to derive a general result on the relationship between Nash Implementation and NEDS Implementation because: A) There might be some SCC that are implementable in NEDS but not in NE because some NEDS sustain "desirable" allocations that are non-sustainable as a NE. B) A SCC might be implementable in NE but not in NEDS, because some NEDS sustain "undesirable" allocations. The first claim is easy to substantiate, because one example is sufficient. This is our Proposition 3 below. The second claim is more difficult to prove because we need to check it for any mechanism implementing a SCC. In fact, we will see, in Propositions 4 and 5 below, that under reasonable conditions, Nash Implementation implies implementation in NEDS and, thus, the problem described in point B) above does not arise.

Let us consider the domain of exchange economies with $n=2$ and two goods. The consumption set of any agent is $\mathbb{R}_{+} \times \mathbb{R}$. Let $\omega_{i} \gg 0$ be the endowment of agent $i=1,2$. Let $\omega_{1}+\omega_{2}=\omega$. Let $x_{i}$ be the consumption of agent $i$, and $x_{i}^{r}$ be the consumption of commodity $r=1,2$ by agent $i=1,2$. The set $A$ reads as follows:

$$
A=\left\{\left(x_{1}, x_{2}\right) \in\left(\mathbb{R}_{+} \times \mathbb{R}\right)^{2}: x_{1}+x_{2} \leq \omega\right\}
$$

Let $E^{q s l}$ be the class of economies with utility functions of the form

$$
u_{i}=v_{i}\left(x_{i}^{1}\right)+x_{i}^{2}
$$

where $v_{i}()$ is smooth and strictly concave. In $E^{q s l}$, all Pareto efficient, scale invariant, and symmetric solutions to the bargaining problem (e.g., Nash 1950; Shapley 1953; Raiffa 1953; Kalai-Smorodinsky 1975, etc.) coincide. This solution (in utility terms) is called the standard solution. Define a SCC $N: E^{q s l} \rightarrow A$ such that for each $e \in E^{q s l}, N(e)$ yields allocations in which utilities are those prescribed by the standard solution. At this point it may be useful to recall that if a SCC is implementable in NE it must be Monotonic, as defined below.

Preferences in $e^{\prime}$ are a monotonic transformation of preferences in $e$ at allocation $a$, denoted by $e^{\prime} \in M T(e, a)$ if

$$
u_{i}(a, e) \geq u_{i}(b, e) \Rightarrow u_{i}\left(a, e^{\prime}\right) \geq u_{i}\left(b, e^{\prime}\right), i=1, \ldots, n
$$

In words, preferences in $e^{\prime}$ are a monotonic transformation of preferences in $e$ at $a$, if $a$ goes up (or stays constant) in the preference rankings of all agents when we go from $e$ to $e^{\prime}$.

A SCC $\phi$ is Monotonic (or Maskin-Monotonic) if

$$
e^{\prime} \in M T(e, a) \Rightarrow\left\{a \in \phi(e) \Rightarrow a \in \phi\left(e^{\prime}\right)\right\} .
$$


Maskin monotonicity says that if $a$ was desired by the planner as an allocation in $e$ and we consider a new economy $e^{\prime}$ in which $a$ goes up in the ranking, $a$ should also be desired in $e^{\prime}$. This property is necessary for Nash Implementation (NI) and, in addition with No Veto Power (defined below), it is sufficient for NI with more than two agents (see, e.g., Jackson 2001).

It is clear now that $N($ ) does not satisfy Maskin Monotonicity and, thus, it is not NI. However, we will show that this solution is implementable in NEDS by a Demand Mechanism. In this kind of mechanism (first introduced by Sjöström 1996) $M_{1}=M_{2}=A$. Let $m_{i}=\left(m_{i i}, m_{i j}\right)$, where $m_{i j}=\left(m_{i j}^{1}, m_{i j}^{2}\right)$ is interpreted as the consumption of agent $j$ suggested by agent $i$ of goods 1 and 2 .

We assume that in a demand mechanism, it is decent to ask for something that does not decrease the utility that the other person can obtain in the absence of an agreement. Note that it is this proposal, and not the resulting allocation, what is required to belong to $D_{i}\left(\mathcal{M}, e, m_{-i}\right)$, i.e., no agent makes a proposal that "pushes the other agent against the wall". There are several interpretations of this. On the one hand, it may reflect the fact that any proposal that is not individually rational for somebody will not be taken seriously. On the other hand, there is a moral justification: it embodies the idea that is morally acceptable to seek one's own pleasure as long as it does not hurt others. A decent proposal therefore is one that does not decrease the welfare that others can obtain in the absence of an agreement. Formally,

A strategy profile $m=\left(m_{i}, m_{j}\right) \in M$ is decent in economy $e=\left(u_{1}, u_{2}\right)$, if for all $i, j=1,2, u_{j}\left(m_{i j}\right) \geq u_{j}\left(\omega_{j}\right)$.

Suppose that $D(\mathcal{M}, e) \subseteq \mathbb{R}_{++}^{4}$ (goods are essential). Then, we obtain the following result:

Proposition 3. Let $E \subseteq E^{s q l}$. Then $N$ can be implemented in NEDS by a demand mechanism with outcome function $F\left(m_{1}, m_{2}\right)=\frac{m_{1}+m_{2}}{2}$.

Proof. First, let us review some well-known facts. Pareto Efficient allocations are the solution to the following program: $i=1,2$

$$
\left(P_{i} 1\right)\left\{\begin{array}{l}
\operatorname{Max} v_{i}\left(x_{i}^{1}\right)+x_{i}^{2}, \\
\text { s.t. } v_{j}\left(\omega^{1}-x_{i}^{1}\right)+\omega^{2}-x_{i}^{2} \geq \bar{u}_{j}, j \neq i
\end{array}\right.
$$

Where $\bar{u}_{j}$ is an arbitrary constant. Because utility functions are strictly increasing, the restriction is binding. Since goods are essential, the solution is interior as long as $\bar{u}_{j}$ is larger than or equal to $u_{j}\left(\omega_{j}\right)$. Because utility functions are strictly concave, the solution is unique and the first order conditions (FOC) for $\left(P_{i} 1\right)$ are necessary and sufficient. They read as follows (derivatives are denoted by primes)

$$
\begin{aligned}
v_{i}^{\prime}\left(x_{i}^{1}\right) & =v_{j}^{\prime}\left(x_{j}^{1}\right) \\
v_{j}\left(\omega^{1}-x_{i}^{1}\right)+\omega^{2}-x_{i}^{2} & =\bar{u}_{j} \\
x_{j}^{r} & =\omega^{r}-x_{i}^{r}, r=1,2
\end{aligned}
$$

In particular, if we let $\bar{u}_{j}=u_{j}\left(\omega_{j}\right)$, we have the following program:

$$
\left(P_{i} 2\right)\left\{\begin{array}{l}
\operatorname{Max} v_{i}\left(x_{i}^{1}\right)+x_{i}^{2}, \\
\text { s.t. } v_{j}\left(\omega^{1}-x_{i}^{1}\right)+\omega^{2}-x_{i}^{2} \geq u_{j}\left(\omega_{j}\right), i=1,2, j \neq i
\end{array}\right.
$$


Let $\left(x_{i i}^{*}, x_{i j}^{*}\right)$ be the unique solution of $\left(P_{i} 2\right), i, j=1,2$. As a consequence of quasilinearity, the Pareto frontier is linear, and the Standard Solution can be expressed as follows:

$$
N(e)=\left[\frac{x_{11}^{*}+x_{21}^{*}}{2}, \frac{x_{12}^{*}+x_{22}^{*}}{2}\right] .
$$

Let $\left(m_{1}, m_{2}\right)$ be a NEDS. Thus, for given $m_{j}, m_{i}$ must solve the following program:

$$
\left(P_{i} 3\right)\left\{\begin{array}{l}
\operatorname{Max} v_{i}\left(\frac{m_{i i}^{1}+m_{j i}^{1}}{2}\right)+\frac{m_{i i}^{2}+m_{j i}^{2}}{2} \\
\text { s.t. } v_{j}\left(\omega^{1}-m_{i i}^{1}\right)+m_{i j}^{2}=u_{j}\left(\omega_{j}\right) .
\end{array}\right.
$$

Taking into account that proposals must be feasible, FOC for $\left(P_{i} 3\right), i=1,2$ are the following:

$$
\begin{aligned}
& v_{1}^{\prime}\left(\frac{m_{11}^{1}+\omega^{1}-m_{22}^{1}}{2}\right)=v_{2}^{\prime}\left(\omega^{1}-m_{11}^{1}\right) \\
& v_{2}^{\prime}\left(\frac{m_{22}^{1}+\omega^{1}-m_{11}^{1}}{2}\right)=v_{1}^{\prime}\left(\omega^{1}-m_{22}^{1}\right) \\
& v_{j}\left(\omega^{1}-m_{i i}^{1}\right)+\omega^{2}-m_{i i}^{2}=u_{j}\left(\omega_{j}\right), i \neq j=1,2 .
\end{aligned}
$$

We are now prepared for the proof.

i) The allocation $N(e)$ can be sustained as a NEDS. Let $m_{i}^{*}=\left(x_{i i}^{*}, x_{i j}^{*}\right)$ for $i, j=1,2$. These messages clearly yield the desired allocation. Moreover, they are a NEDS, because they fulfill the FOC of a NEDS and under our assumptions, FOC are sufficient.

ii) Any NEDS yields $N(e)$. Consider again FOC in a NEDS.

Since $v_{i}()^{\prime}$ s are strictly concave, $v_{i}^{\prime}()^{\prime}$ s are invertible. Denoting the inverse of $v_{i}^{\prime}\left(\right.$ ) by $v_{i}^{\prime-1}()$ and letting $y \equiv m_{11}^{1}-m_{22}^{1}$ the first two equations above can be written as

$$
\begin{aligned}
& v_{2}^{\prime-1}\left(v_{1}^{\prime}\left(\frac{y+\omega^{1}}{2}\right)\right)=\omega^{1}-m_{11}^{1}, \\
& v_{1}^{\prime-1}\left(v_{2}^{\prime}\left(\frac{\omega^{1}-y}{2}\right)\right)=\omega^{1}-m_{22}^{1} .
\end{aligned}
$$

Subtracting (3.2) from (3.1) above we get

$$
y=v_{1}^{\prime-1}\left(v_{2}^{\prime}\left(\frac{\omega^{1}-y}{2}\right)\right)-v_{2}^{\prime-1}\left(v_{1}^{\prime}\left(\frac{\omega^{1}+y}{2}\right)\right) .
$$

$v_{i}^{\prime-1}\left(v_{j}^{\prime}()\right),(i \neq j=1,2)$ is an increasing function because it is the composition of two decreasing functions. Thus, the right-hand side of the above equation is decreasing on $y$. Since the left-hand side is increasing on $y$, there is only one value of $y$ that satisfies this equation. Now from equations (3.1) and (3.2) above we obtain that $m_{11}^{1}$ and $m_{22}^{1}$ are also unique, and, given that $v_{j}\left(\omega^{1}-m_{i i}^{1}\right)+m_{i j}^{2}=u_{j}\left(\omega_{j}\right), i \neq$ $j=1,2$, we find that $m_{12}^{2}$ and $m_{21}^{2}$ are also unique. Finally, by feasibility, $m_{21}^{1}=$ 
$\omega^{1}-m_{22}^{1}, m_{12}^{1}=\omega^{1}-m_{11}^{1}, m_{11}^{2}=\omega^{2}-m_{12}^{2}$ and $m_{22}^{2}=\omega^{2}-m_{21}^{2}$. Therefore, the NEDS is unique and, from $i$ ) above, it yields the desired allocation.

Proposition 3 above can be proven for an arbitrary number of goods if the utility function is additively separable in all goods. ${ }^{9}$ Unfortunately, relaxing quasilinearity has the implication that no sub-correspondence of the Pareto correspondence can be implemented in NEDS by a demand mechanism (see Corchón and Herrero 1995, pp. 27-29 for more on this).

We now present a condition necessary for implementation in NEDS.

Remark 3.1 If $\phi$ is implementable in NEDS by means of a mechanism $\mathcal{M}$, then, the following condition holds:

$$
\begin{aligned}
& \left\{e^{\prime} \in M T(e, a), D\left(\mathcal{M}, e^{\prime}\right) \subseteq D(\mathcal{M}, e), D\left(\mathcal{M}, e^{\prime}\right) \cap D N S(\mathcal{M}, e) \neq \emptyset\right\} \Rightarrow \\
& \quad \Rightarrow\left\{a \in \phi(e) \Rightarrow a \in \phi\left(e^{\prime}\right)\right\} .
\end{aligned}
$$

Proof. Since $\phi$ is implementable in NEDS, $\exists m \in D(\mathcal{M}, e)$ such that $g(m) \in \phi(e)$. Then, $m \in D\left(\mathcal{M}, e^{\prime}\right)$. Since $a$ is now more (or equally) preferred by all agents, $m \in D N S(\mathcal{M}, e) \Rightarrow m \in D N S\left(\mathcal{M}, e^{\prime}\right)$. Since $\phi$ is implementable it must be that $g(m) \in \phi\left(e^{\prime}\right)$.

It is clear that if $D(\mathcal{M}, e)=M, \forall e \in E$ the above condition reduces to Maskin Monotonicity, i.e.

$$
e^{\prime} \in M T(e, a) \Rightarrow\left\{a \in \phi(e) \Rightarrow a \in \phi\left(e^{\prime}\right)\right\} .
$$

Another case in which the above condition reduces to Maskin Monotonicity is when decency constraints consist only of the sets $d_{i}(e)^{\prime}$ s, and $d_{i}(e)=d_{i}\left(e^{\prime}\right)$, for all $i, e, e^{\prime}$. An example of the latter condition in the framework of an exchange economy is when the set $d_{i}(e)$ consists of the allocations in which the consumption of $i$ is not strictly larger than $i$ 's endowments. Unfortunately, the necessary condition in the remark above implies that, in order to verify it, we have to know what the sets $D\left(\mathcal{M}, e^{\prime}\right)$ look like.

We now concentrate on the study of sufficient conditions for a SCC to be implementable in NEDS. We will see that with slight modifications, sufficient conditions for Nash implementation also become sufficient conditions for implementation in NEDS. Let $a_{i}^{*}(e) \in \arg \max u_{i}(a, e), a \in d_{i}(e)$ be a top allocation for $i$ in the set of decent allocations.

A SCC $\phi$ satisfies Strong No Veto Power $(S N V P)$ if

$$
\left\{a_{i}^{*}(e)=a_{j}^{*}(e) \text { for, at least, } n-2 \text { agents }\right\} \Rightarrow a_{i}^{*}(e) \in \phi(e) .
$$

If $d_{i}(e)=A \forall i$, we have the standard definition of No Veto Power, except that, here, we need $n-2$ agents (and not $n-1$ ) to agree on some allocation. In the domain of exchange economies (assuming strictly increasing preferences) with $n>2$, No Veto Power is vacuously satisfied because $a_{i}^{*}(e) \neq a_{j}^{*}(e)$ for all $i, j$. In the case

\footnotetext{
9 Notice that in the case of Proposition 3, the outcome function is continuous and the best reply is single valued because payoff functions are strictly concave on messages.
} 
of Strong No Veto Power, the property depends on sets $d_{i}(e)$ 's. For instance, in the domain of exchange economies (assuming strictly increasing preferences) with $n>3$, it is easy to see that if the dimension of the sets $d_{i}(e)^{\prime} s$ equals the dimension of $A$, Strong No Veto Power holds vacuously. We think of this property as being more plausible the larger $n$ is.

As a final piece of notation, let $L_{i}(a, e)$ be the set of allocations that are not preferred by $i$ to $a \in A$ according to the utility function $u_{i}\left(\right.$, e), i.e., $L_{i}(a, e)$ is the lower contour set of $i$ at allocation $a$.

The mechanism that we will use (denoted by $\mathcal{M})$ is standard in Nash implementation: A message from $i, m_{i}=\left(e_{i}, a_{i}, k_{i}\right) \in E \times A \times \Re$. The outcome function is defined by the following three rules:

Rule I: If $m$ is such that $m_{i}=m_{j}=\left(e_{i}, a_{i}, k_{i}\right), \forall i, j$, and $a_{i} \in \phi(e), g(m)=a_{i}$.

Rule II: If $m$ is such that $m_{i}=m_{j}=\left(e_{i}, a_{i}, k_{i}\right), a_{i} \in \phi(e), \forall i, j$, except $k$, $g(m)=a_{k}$ if $a_{k} \in L_{k}\left(a_{i}, e_{i}\right) \cdot g(m)=a_{i}$ otherwise.

Rule III: In any other case, $g(m)=a_{j}$ where $k_{j} \geq k_{i} \forall i$ and if $k_{j}=k_{h}, j<h$.

This mechanism has been extensively discussed in the literature, see, e.g. Jackson (2001). In this mechanism, we impose the following properties on the set of decent messages. From now on, let $e$ denote the true economy:

$\alpha$ ) Suppose $\tilde{m}_{i}=(e, a, k)$ with $a \in \phi(e)$. Then $\tilde{m}_{i} \in D_{i}\left(\mathcal{M}, e, \tilde{m}_{-i}\right)$, (to tell the truth and to announce an allocation prescribed by the SCC at the corresponding economy, is a decent message if everybody says so).

$\beta)$ Suppose $m_{i}=m_{j}=\left(e_{i}, a_{i}, k_{i}\right) \forall i, j$, except $h$. Then if $a \in L_{h}\left(a_{i}, e_{i}\right)$ $(e, a, k) \in D_{h}\left(\mathcal{M}, e, m_{-h}\right)$ (it is decent for $h$ to ask for any allocation that, according to everybody else, decreases her utility).

For the next property we need a new definition: Let

$U M_{i}\left(a, e, m_{-i}\right)=\left\{m_{i} \in M_{i} / u_{i}\left(g\left(m_{i}, m_{-i}\right), e\right)>u_{i}(a, e), g\left(m_{i}, m_{-i}\right) \in d_{i}(e)\right\}$.

$U M_{i}\left(a, e, m_{-i}\right)$ is the set of messages of $i$ that, given the messages of the other agents, yield a decent allocation preferred by $i$ to $a$ in economy $e$. Let $\check{m}_{-i}$ be such that there are, at least two agents, say $j$ and $h$ with $m_{j} \neq m_{h}$.

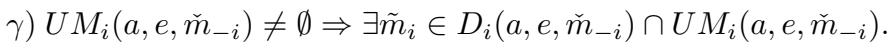

This property says that if, at least, two agents send conflicting reports and $i$ can improve her payoff by means of some messages, at least one of these messages is decent. In other words, if there is a conflict -and agent $i$ is neither responsible for it, nor can it be fixed by her-, $i$ is entitled to a payoff improvement, if she can get it. The interpretation is that all agents are supposed to send identical messages and once this norm is broken it is decent for other agents to seek improvements. Notice that the improvement may be small: For instance in the domain of exchange economies, it might consist of an infinitesimal increase in the consumption of $i$ of some commodity. 
Finally, two remarks. One, that the mechanism is independent of decency requirements, so the planner does not have to known the particular decency requirements of a society in order to implement a certain SCC. Two, notice that conditions $\alpha-\beta-\gamma$ refer to different parts of the mechanism, and thus, there is no incompatibility among them.

Proposition 4. Under conditions $\alpha, \beta$ and $\gamma$, if $n>3$, the above mechanism implements in NEDS any SCC satisfying Monotonicity and Strong No Veto Power.

Proof.

Step 1: If $a \in \phi(e), \exists m \in D N S(\mathcal{M}, e)$ such that $g(m)=a$. Let $m=$ $\left(m_{1}, \ldots, m_{n}\right)$ with $m_{i}=(e, a, 1)$. By $\left.\alpha\right)$, this is a decent strategy profile. Any possible deviation can only drive the outcome to Rule II) above and, clearly, no agent can improve with such a deviation.

Step 2: If $m \in D N S(\mathcal{M}, e), g(m) \in \phi(e)$. We have three possible cases:

i) Suppose that $m$ is such that we are in Rule I). If $e \in M T\left(e^{\prime}, a\right)$, by Monotonicity, $a \in \phi\left(e^{\prime}\right) \Rightarrow a \in \phi(e)$, and we are done. If $e \notin M T\left(e^{\prime}, a\right)$,there is an allocation, say $\tilde{a}$, and an agent, say $i$, such that $u_{i}(\tilde{a}, e)>u_{i}\left(a^{\prime}, e\right)$ and $u_{i}\left(\tilde{a}, e^{\prime}\right)<u_{i}\left(a^{\prime}, e^{\prime}\right)$. By $\left.\beta\right), m_{i}=(e, \tilde{a}, 1)$ is a decent message that, by Rule II), yields $\tilde{a}$, which is a contradiction.

ii) Suppose that $m$ is such that we are in Rule II). Let $a$ be the resulting allocation. Let the agent who sends a message different from the others be denoted by $i$. Thus $m_{i}=\left(e_{i}, a_{i}, k_{i}\right) \neq m_{j}=\left(e_{j}, a_{j}, k_{j}\right)$. If $U M_{h}\left(a, e, \check{m}_{-h}\right) \neq \emptyset$ some $h \neq i, j$, by $\gamma)$ there is a message, say $m_{h} \in D_{h}\left(a, e, \check{m}_{-h}\right) \cap U M_{i}\left(a, e, \check{m}_{-h}\right)$ which yields an allocation preferred by $h$ to $a$, and this is a contradiction. Suppose now that $U M_{h}\left(a, e, \check{m}_{-h}\right)=\emptyset$ for all $h \neq i, j$. But these agents could have obtained any decent allocation, say $a_{h}$ by sending a message $m_{h}=$ $\left(e_{h}, a_{h}, k_{h}\right)$ with a large enough $k_{h}$. Thus, it must be that $a$ is a top allocation in the set of decent allocations for, at least, all agents minus $i$ and $j$. Again, by Strong No Veto Power, $a \in \phi(e)$.

iii) Suppose that $m$ is such that we are in Rule III) above. By an identical reasoning as in ii) above, we can show that either $g(m)$ is a top decent allocation for $n-2$ agents (and thus, $g(m) \in \phi(e)$ ) or $m$ is not a NEDS

As we remarked before, the sets $D(\mathcal{M}, e)$ can be understood as being determined by culture. Thus, different cultures will produce different sets $D(\mathcal{M}, e)$ 's and, consequently some SCC could be implemented in NEDS in some societies but not in others. ${ }^{10}$ We now provide a different set of axioms defining the sets $D(\mathcal{M}, e)$ 's and we will show that a stronger result than Proposition 4 is obtained.

A message for $i, m_{i}=\left(e_{i}, k_{i}\right) \in E \times \Re$. The outcome function is defined by the following two rules:

Rule I: If $m$ is such that $e_{i}=e_{j}, \forall i, j, g(m)=\phi\left(e_{j}\right)$.

${ }^{10}$ In this case NEDS is not strict since there are several best replys when everybody else announces the true economy and, moreover, the outcome function is not continuous. The first problem can be fixed by penalizing the dissident with a small fine. However the problem of implementing in NE a SCC by a continuous mechanism is still open. 
Rule II: In any other case, $g(m)=\phi\left(e_{j}\right)$ where $k_{j} \geq k_{i} \forall i, k_{j}=k_{h}$,if $j<h$.

In this mechanism, we have new decency requirements that are similar to $\alpha-$ $\beta-\gamma$. They are as follows.

$\alpha 1)$ Suppose $\tilde{m}_{i}=(e, k)$. Then $\tilde{m}_{i} \in D_{i}\left(\mathcal{M}, e, \tilde{m}_{-i}\right)$, (to tell the truth is a decent message if everybody says so).

$\beta 1)$ Suppose $m_{i}=m_{j}=\left(e_{i}, k_{i}\right) \forall i, j$, except $h$. Then if $e_{i} \neq e,(e, k) \in$ $D_{h}\left(\mathcal{M}, e, m_{-h}\right)$ (it is decent for $h$ to tell the truth if everybody else is not telling the truth about the economy).

$\beta 2)$ Suppose $m_{i}=m_{j}=\left(e, k_{i}\right) \forall i, j$, except $h$. Then if $e_{h} \neq e, m_{h}=\left(e_{h}, k_{h}\right), \notin$ $D_{h}\left(\mathcal{M}, e, m_{-h}\right)$ (it is not decent for $h$ not to tell the truth if everybody else is telling the truth about the economy).

1) $U M_{i}\left(a, e, \check{m}_{-i}\right) \neq \emptyset \Rightarrow \exists \tilde{m}_{i} \in D_{i}\left(a, e, \check{m}_{-i}\right) \cap U M_{i}\left(a, e, \check{m}_{-i}\right)$ where $\check{m}_{-i}$ is such that there are, at least two agents, say $j$ and $h$ with $m_{j} \neq m_{h}$ and

$$
\begin{aligned}
& U M_{i}\left(a, e, m_{-i}\right) \\
& =\left\{m_{i} \in M_{i} / u_{i}\left(g\left(m_{i}, m_{-i}\right), e\right)>u_{i}(a, e), g\left(m_{i}, m_{-i}\right) \in \phi\left(e^{\prime}\right), e^{\prime} \in E\right\} .
\end{aligned}
$$

$U M_{i}\left(a, e, m_{-i}\right)$ is now the set of messages from $i$ that, given the messages of the other agents, yield an allocation that belongs to $\phi\left(e^{\prime}\right)$, some $e^{\prime} \in E$ and which is preferred by $i$ to $a$ in economy $e$.

Conditions $\alpha 1$ and $\gamma 1$ are adaptations of $\alpha$ and $\gamma$ to the new mechanism. Conditions $\beta 1-\beta 2$ are a stronger than $\beta$. $\beta 1$ recalls the situation in Henryk Ibsen's play "An Enemy of the People" in which the unsanitary condition of a village is exposed by a doctor, in spite of the silence of everybody else. $\beta 2$ is appropriate when $n$ is large, because nobody wants to confront a crowd when she has lied and the rest have not, think of the consequences of cheating in your tax form when everybody else has been truthful.

Instead of SNVP, we now have the following condition: Let

$$
S L_{i}(e)=\left\{a \in A / u_{i}(a, e) \geq u_{i}\left(a^{\prime}, e\right), a^{\prime} \in \phi\left(e^{\prime}\right), e^{\prime} \in E\right\} .
$$

In words, $S L_{i}(e)$ is the set of allocations that maximize $i$ 's utility in economy $e$ over all the allocations chosen by $\phi()$ (this is reminiscent of $i$ being a Stackelberg leader, hence the acronym). Now we have the following definition:

A SCC $\phi$ satisfies Very Strong No Veto Power (VSNVP) if

$$
\left\{a_{i} \in S L_{i}(e) \text { for, at least, } n-2 \text { agents }\right\} \Rightarrow a_{i}(e) \in \phi(e) .
$$

Once again, this is a condition that is plausible when $n$ is large. It is easy to see that in exchange economies with $n>2$ and with "sufficiently rich" $E$, VSNVP is vacuously satisfied. We will also assume that if $u_{i}(a, e)=u_{i}(b, e), \forall i, a=b$, i.e., if all agents think that allocations $a$ and $b$ are indifferent, these allocations are, in fact, the same allocation. This assumption forbids indifference curves that are thick or parallel to each other. It is satisfied in exchange economies with convex 
preferences in which at least one agent has strictly convex preferences. Now we have the following:

Proposition 5. If $n>3$, the above mechanism implements any single-valued Pareto Efficient SCC satisfying VSNVP in NEDS.

Proof.

Step 1: If $a \in \phi(e), \exists m \in D N S(\mathcal{M}, e)$ such that $g(m)=a$. Let $m=$ $\left(m_{1}, \ldots, m_{n}\right)$ with $m_{i}=(e, 1)$. By $\alpha 1$, this is a decent strategy profile and by $\beta 2$ any deviation to Rule II is not decent.

Step 2: If $m \in D N S(\mathcal{M}, e), g(m) \in \phi(e)$. We have two possible cases:

i) Suppose that $m$ is such that we are in Rule I). Let $m_{i}=\left(e^{\prime}, k^{\prime}\right), \forall i$. If $e^{\prime}=e$, we are done. If $e^{\prime} \neq e$, we have two possibilities: If $u_{i}(\phi(e), e)=u_{i}\left(\phi\left(e^{\prime}\right), e\right), \forall i$, $\phi(e)=\phi\left(e^{\prime}\right)$ and we are done. If not, since $\phi()$ is Pareto Efficient, there is, at least one agent, say $i$, for whom $u_{i}(\phi(e), e)>u_{i}\left(\phi\left(e^{\prime}\right), e\right)$. But for this agent, by $\beta 1$, it is decent to send a message like $m_{i}=\left(e, k_{i}\right)$ with $k_{i}>k^{\prime}$ for which her payoff improves, which is a contradiction.

ii) Suppose that $m$ is such that we are in Rule II). Let $a$ be the resulting allocation. Let $i$ and $j$ be such that $m_{i}=\left(e_{i}, k_{i}\right) \neq m_{j}=\left(e_{j}, k_{j}\right)$. If $U M_{h}\left(a, e, \check{m}_{-h}\right) \neq \emptyset$ some $h \neq i, j$, by $\left.\gamma\right)$ there is a message, say $m_{h} \in$ $D_{h}\left(a, e, \check{m}_{-h}\right) \cap U M_{i}\left(a, e, \check{m}_{-h}\right)$ which yields an allocation preferred by $h$ to $a$, and this is a contradiction. Therefore, it must be that $U M_{h}\left(a, e, \check{m}_{-h}\right)=\emptyset$ for all $h \neq i, j$. Thus it must be that $a$ is a top allocation for, at least, all agents minus $i$ and $j$ in the set $S L_{h}(e)$. Again, by VSNVP, $a \in \phi(e)$.

One implication of Propositions 4 and 5 is as follows: Take a space of economies for which Very Strong No Veto Power, Strong No Veto Power and No Veto Power hold vacuously (i.e., exchange economies with $n>3$ ). If a SCC defined in this space of economies is NI, it is also implementable in NEDS. Therefore, the consideration of decency constraints enlarges the set of implementable SCC.

To sum up, then, under reasonable conditions, NI implies implementation in NEDS but the converse is not true. The latter result is explained by the fact that to "sustain" an allocation selected by the SCC by means of a NE is always possible because to tell the truth is always a NE. Thus, if a SCC is not NI it is because there is a Nash Equilibrium (NE) yielding allocations that do not belong to the SCC for the corresponding economy. Maskin Monotonicity is used to "kill" these unwanted allocations. We have provided an example (see Proposition 3) in which decency constraints -not Maskin-Monotonicity- are used to "kill" these unwanted allocations.

\section{Final comments}

In this paper, we have studied how decency constraints affect resource allocations in some particular environments. Our results can be understood from a twofold perspective. From the bargaining point of view, we study the non-cooperative foundations of two specific cases. Instead of looking for reasonable axioms that a solution 
must satisfy, we look for simple procedures and identify the solutions as (decent) non-cooperative equilibria. From the implementation point of view, our paper is a (possibly crude) formalization of the idea that, in addition to resource and incentive compatibility constraints, organizations face fairness constraints: people must perceive the behavior of others as being fair, or at least decent (see van Damme 1986 for an alternative formalization of this idea). If they do not, they might behave in unexpected, outrageous ways: The history of the world abounds with this sort of behavior - industrial sabotage, etc. -. Experimental evidence that fairness matters in bargaining also abounds (see, e.g., Roth 1995, pp. 287-288). We have shown that, in certain cases, decent behavior makes the planner's job easier. For instance, the standard solution to bargaining problems cannot be implemented in Nash Equilibrium, but it can be implemented in Nash Equilibrium in Decent Strategies. Another possibility, not explored in this paper, is that the mechanism must be perceived as being fair. This would tie the hands of the designer, because some mechanisms could be objected to on the grounds that the rules of the game are not fair. From an analytical point of view, the problem would be similar to that of Chakravorti, Corchón and Wilkie, (1994), even though the motivation in this paper is very different, i.e., the inability of the planner to commit herself to certain allocations.

\section{References}

Anbarci, N., Yi, G. (1992) A Meta Allocation Mechanism in Cooperative Bargaining. Economics Letters 38: 175-179

Aumann, R., Maschler, M. (1988) Game Theoretic Analysis of a Bankruptcy Problem from the Talmud. Journal of Economic Theory 36: 195-213

Chakravorti, B., Corchón, L., Wilkie, S. (1994) Credible Implementation. W. P. of the University of Alicante. Games and Economic Behavior (forthcoming)

Chun, Y. (1989) A Noncooperative Justification for Egalitarian Surplus Sharing. Mathematical Social Sciences 17: 245-261

Corchón, L., Herrero, C. (1995) A Decent Proposal. A Discusión, WP-AD 95-25. Universidad de Alicante

Dagan, N., Volij, O. (1993) The Bankruptcy Problem: A Cooperative Bargaining Approach. Mathematical Social Sciences 26: 287-297

Farber, H.S. (1981) Splitting-the-difference in Interest Arbitration. Industrial and Labor Relations Review 35: 70-77

Herrero, C. (2003) Equal Awards vs Equal Losses: Duality in Bankruptcy. In: Sertel, M., Koray, S. (eds.) Advances in Economic Design. Springer, Berlin Heidelberg New York

Jackson, M. (2001) A Crash Course in Implementation Theory. Social Choice and Welfare 18(4): 655708

Kalai, E., Smorodinsky, M. (1975) Other Solutions to Nash Bargaining Problem. Econometrica 43: $513-518$

Nash, J. (1950) The Bargaining Problem. Econometrica 28: 155-162

O’Neill, B. (1982) A Problem of Rights Arbitration in the Talmud. Mathematical Social Sciences 2: 345-371

North, D. (1989) Comments 2. In: Stiglitz, J. et al., Heertjee, A. (eds) The Economic Role of the State. Basil Blackwell, Oxford

Prelec, D., Herrstein, H. (1991) Preferences or Principles: Alternative Guidelines for Choice. In: Zeckhauser, R.J. (ed) Strategy and Choice. The MIT Press, Cambridge, Mass

Rabin, M. (1993) Game Theory and Fairness. American Economic Review 83: 1281-1302 
Raiffa, H. (1953) Arbitration Schemes for Generalized Two-Person Games. In: Khun H.W., Tucker, A.W. (eds) Contributions to the Theory of Games II (Annals of Mathematical Studies 28). Princeton University Press, Princeton

Roth, A. (1995) Bargaining Experiments. In: Kagel, J.H., Roth, A. (eds) The Handbook of Experimental Economics. Princeton University Press, New Jersey

Sen, A. (1983) Liberty and Social Choice. Journal of Philosophy 80: 5-28

Sen, A. (1993) Internal Consistency of Choice Econometrica 61: 495-521

Shapin, S. (1994) A Social History of Truth. The University of Chicago Press, Chicago

Shapley, L.S. (1953) A Value for n-Person Games. In: Khun, H.W., Tucker, A.W. (eds) Contributions to the Theory of Games II (Annals of Mathematical Studies 28). Princeton University Press, Princeton

Sjöström, T. (1996) Implementation by Demand Mechanisms. Economic Design 1: 343-354

Stevens, C. (1966) Is Compulsory Arbitration compatible with Bargaining? Industrial Relations 5: $38-52$

Thomson, W. (1995) Axiomatic Analysis of Bankruptcy and Taxation Problems: A Survey. Mimeo, University of Rochester

Van Damme, E. (1986) The Nash Bargaining Solution is Optimal. Journal of Economic Theory 38: $78-100$ 